EPiC Series in Computing
Volume 62, 2019, Pages 206-212
SUMO User Conference 2019

\title{
Controlling a real-world intersection with connected vehicle information provided by CAMs (Cooperative Awareness Messages)
}

\author{
Daniel Wesemeyer and Jan Trumpold \\ 1 Institute of Transportation Systems, German Aerospace Center (DLR) \\ daniel. wesemeyer@dlr.de \\ 2 Institute of Transportation Systems, German Aerospace Center (DLR) \\ jan.trumpold@dlr.de
}

\begin{abstract}
The project MAVEN (see https://www.maven-ts.eu), funded by the European Commission, aims at developing a system for infrastructure-assisted platoon organization and green phase negotiation for automated connected vehicles (ACVs). Vehicle-to-Everything (V2X) communication protocols are hereby used for the insertion of vehicles into a traffic simulation of a real-world intersection. Until now, real world traffic could be inserted into a simulation through stationary detectors, for example magnet field sensors, induction loops, cameras, radar etc. The downside of this detection method is that only momentary information can be obtained and e.g. the behavior of the vehicles approaching an intersection can only be approximated. ACVs however continuously broadcast their positions and speeds via CAMs. Detecting vehicles though these messages leads to a more realistic representation of the vehicle's driving behavior. The current paper describes how CAMs are used to place and move ACVs inside the simulation of a real-world intersection in Braunschweig with the traffic simulation SUMO (Simulation of Urban Mobility). Furthermore, it describes an approach to how these continuously detected vehicles could be further used as control units. Since the positions and speeds of ACVs are synchronized with the real-world behavior, they can be used to adjust the simulated upstream movements and positioning of conventional vehicles (CV) to match reality. Until all vehicles are equipped with V2X technology, this approach could enable more realistic simulated traffic flow behavior.
\end{abstract}

\section{Introduction}

Cooperative vehicles are an important part of future ITS (Intelligent Transportation Systems). The exchange of information between vehicles (Vehicle-to-Vehicle, V2V) and vehicles and the road infrastructure (Vehicle-to-Infrastructure, V2I, and Infrastructure-to-Vehicle, I2V) is considered a great enabler for increasing road safety and traffic throughput. Thus, connected, cooperative mobility is a highly-active field of research with a great variety of fields of application, some of which are traffic safety, traffic flow harmonization and adaptive signal control.

As V2X-based technologies concerning motorways and trucks have been extensively investigated, research often concentrates on urban contexts. Of course, traffic safety must be ensured 
in a world of connected - and prospective automated - vehicles. As aforementioned, V2X is considered an opportunity to even increase traffic safety, especially in urban areas where vulnerable road users (i.e. pedestrians and cyclists) are often involved in traffic accidents. Several studies deal with this topic and propose V2X-based systems based on hazard detection and / or automotive assistance [1] [2].

In the field of traffic flow harmonization, dynamic speed advices and platooning are two V2X-based extensions that might help reduce congestion. Platooning minimizes gaps between vehicles and thus reduces passing times of vehicles over intersections. Speed advices can be used to let vehicles pass an intersection without stopping, leading to reduced time losses and emissions due to braking and accelerating [3] [4] [5].

Along with platooning, adaptive signal control in urban areas is another important field of application for V2X technologies. Several algorithms that optimize phase sequences and phase durations based on vehicle position data provided by V2X have been developed and significantly reduce vehicle waiting times compared to conventional adaptive control methods [6][7].

The aforementioned position data of vehicles is transmitted as Cooperative Awareness Message (CAM). This is a standard defined by the European Telecommunications Standards Institute (ETSI). CAM is a basic set of information to make other connected traffic participants and the infrastructure aware of the presence of a traffic object. This message type contains for example information about the object's type (e.g. passenger car, truck, bus etc.) and its current position, heading and speed. Typically, CAMs must at least be broadcasted with 10 $\mathrm{Hz}$ to enable vehicle-vehicle and vehicle-infrastructure cooperation and to avoid crashs [8].

The fact that CAMs provide information about the type, the position and the speed of an object on a regular basis qualifies them as a data input for traffic control methods. Conventional vehicle detection is done through static detectors, e.g. induction loops or magnetic field sensors. These detectors provide data at the moment a vehicle disturbs their magnetic field. This detector data has been used before to insert traffic demand into a traffic simulation coupled with a traffic light controller by adding a vehicle with the maximum free speed at the detector position. The disadvantage of this approach is that the downstream traffic flow behaviour, for example the approach of a red traffic light, can only be approximated by the underlying model. CAMs however, close this data gap since they continuously provide position and speed information which can be fed into the simulation.

In section 2, the approach on processing CAMs will be described. Afterwards, two applications of this algorithm are presented that improve simulated traffic flow behaviour at low penetration rates of CAM sending vehicles. Section 3 describes the real-world test of this approach on an intersection in Braunschweig, Germany. In section 4, summarizes the results and gives a short outlook on future activities.

\section{Methodology}

The method of processing CAM data in a traffic simulation presented in this paper consists of a main part and two extensions. The main part describes how vehicles from various data sources (i.e. CAMs and static detectors) are inserted in the same simulation, the extensions apply the additional data obtained through CAMs to influence the simulated traffic flow.

The procedure of inserting CAM-detected vehicles into a traffic simulation is described in Algorithm 1. The vehicles' driving behaviour should be determined only by position updates they provide, not by the internal logic of the traffic flow model. Thus, all CAM vehicles that were inserted in the previous simulation step need to be removed from the simulation. The removal of the CAM vehicles also might be necessary due to data security issues with V2X 


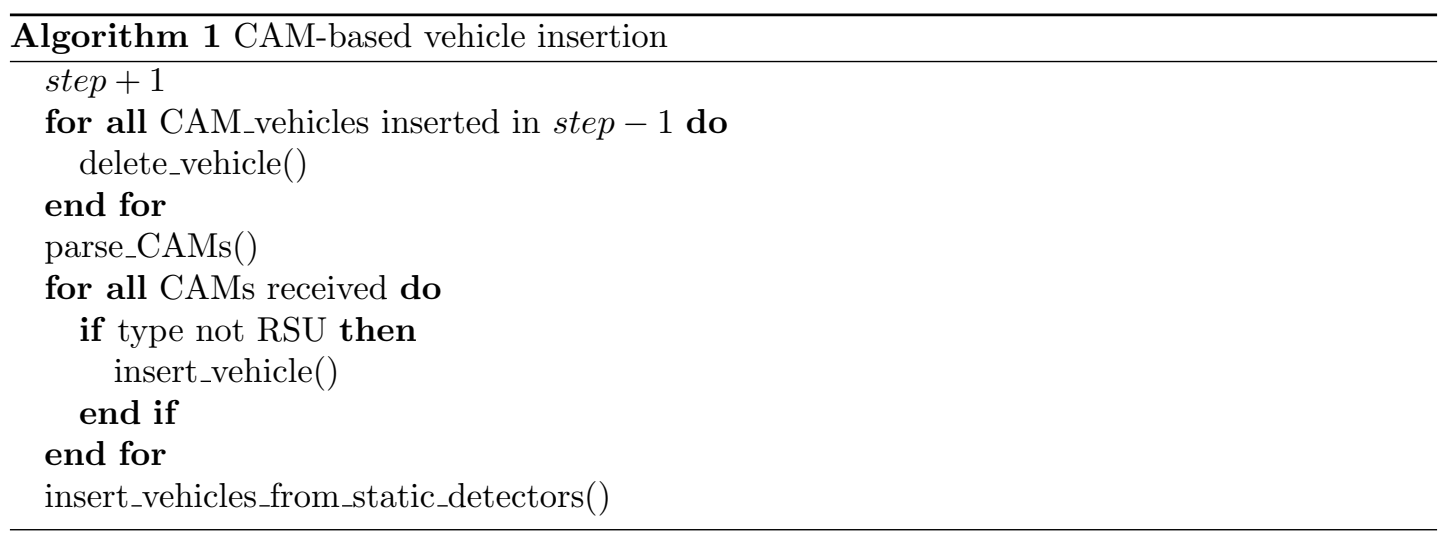

data. Since it would be possible to re-construct single vehicle trajectories on intersections from their CAMs, a possible solution might be to introduce short-term changing identifiers. In case such measures are taken, the deletion of previously detected vehicles would be unavoidable. In the next step, the CAMs received in the current step are parsed. The result is a list of attributes that describe the vehicles' states:

- id: A unique identifier for the vehicle

- type: The vehicle type, i.e. passenger car, taxi, bus etc.

- coord: The position of the vehicle (lat, lon)

- speed: The current longitudinal speed of the vehicle

For each CAM, a type check is made since the definition of CAMs does not only apply to moving objects such as passenger cars but also to Road-Side-Units (RSU). This check ensures that only moving objects are inserted. The vehicles are inserted at their respective positions and assigned the speed values they reported. Afterwards, conventional vehicles that were detected by static detectors (e.g. induction loops) can be inserted. It is important to insert the vehicles in this order since otherwise, they would be detected as conventional vehicles, leading to a loss of information and potentially an over-estimation of traffic demand.

Apart from just using CAMs as additional data input, they can be used to adapt the behaviour of the conventionally detected vehicles. As shown in Figure 1, a CAM vehicle is inserted into the simulation with a reported speed of $40 \mathrm{~km} / \mathrm{h}$. Upstream, a CV is inserted at the detector position with a free speed of $50 \mathrm{~km} / \mathrm{h}$. Without the presence of the CAM vehicle, the $\mathrm{CV}$ would drive downstream the road with free speed. Due to the reduced speed of the CAM vehicle, the CV needs to adapt its speed to $40 \mathrm{~km} / \mathrm{h}$ in order to avoid a collision. This speed adaption of the simulated vehicles leads to a more realistic representation of the edge's traffic flow. The adaption continues upstream as more CVs are inserted.

Another possible field of application of CAMs in a traffic simulation is to use them as detector vehicles for queue lengths in front of a red traffic light (see Fig. 2). In the following, two possible applications are presented. The first approach is an application of an approximation method described by Priemer and Friedrich [9]. A downside of contemporary detection is that sometimes, vehicles passing detectors are not detected which leads to underestimation of the traffic demand. When running an adaptive control algorithm, this can lead to inefficiencies or in the worst case to extremely high waiting times if it occurs on approaches of an intersection 


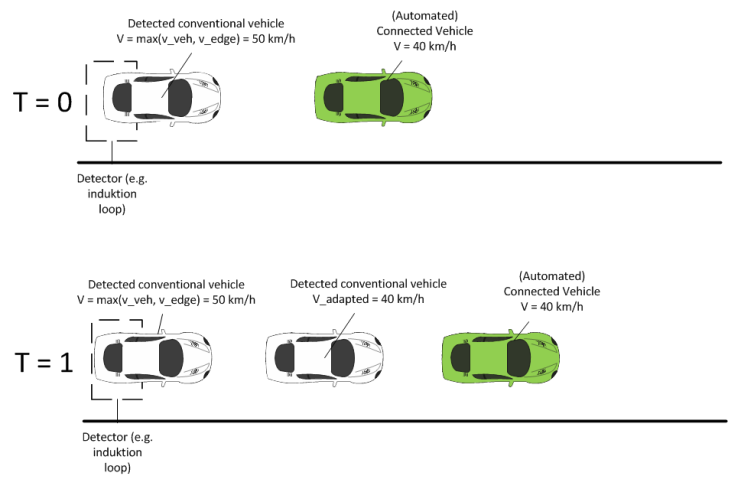

Figure 1: ACV as detector vehicle in a free flow traffic situation.

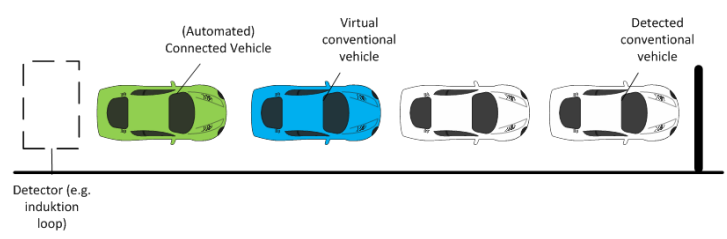

Figure 2: ACV as detector vehicle in a queue in front of a red traffic light.

with an overall low demand. From the stop position of a CAM vehicle we can obtain the minimum length of the queue. If a gap larger than the length of a vehicle occurs between the $\mathrm{CAM}$ vehicle and the last detected $\mathrm{CV}$ or the stop line, we can assume that there could be another vehicle which was not detected previously. By measuring the gap between the CAM vehicle and the last CV in the queue and assuming a standard length per vehicle plus minimum gap to the preceding vehicle, "virtual CVs" could be added to match the demand on the queue. The number of virtual vehicles to be inserted would be $l_{\text {queue_gap }} /\left(l_{\text {vehicle }}+l_{\text {min_gap }}\right)$.

The second approach in the field of queue length correction would be to remove vehicles that passed the traffic light in reality, but did not in the simulation. In reality, drivers sometimes pass traffic lights that just switched to red, either because they were not able to brake or they did not want to stop because of subjective time pressure. However, in the simulation these vehicles in general do not pass red traffic lights because they strictly obey the set of traffic rules given to them ${ }^{1}$.

Note Figure 3 for the description of this approach. In the real world, a CV passed the traffic light shortly after it turned red, the ACV stops in front of the traffic light and sends its position information to the simulation. In the simulation, the underlying rules forced the detected CV stopped in front of the red traffic light, the ACV is enqueued behind it. However, the position sent by the real-world ACV is the same as the position of the CV that (in reality) already passed the intersection. This means, if the position provided by a ACV is the same as the position of a simulated CV, we can assume that the CV already passed the intersection and remove it from the simulation.

Both applications work with low penetration rates since only one ACV is needed per approaching lane. Thus, we have one detector vehicle per lane with which traffic flow and queue

\footnotetext{
${ }^{1}$ This behavior is actually configurable in many traffic simulations like SUMO, but the default setting is that vehicles do not pass red traffic lights.
} 


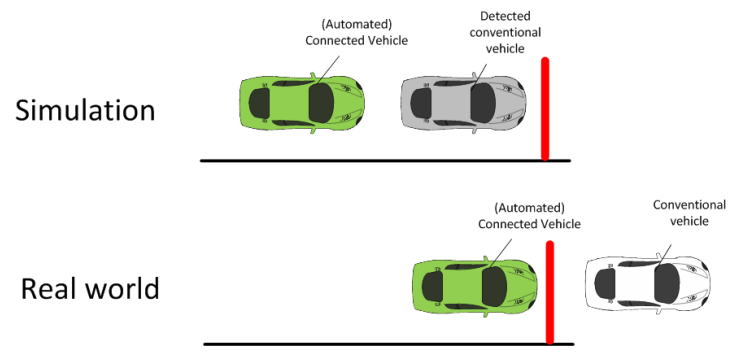

Figure 3: ACV as detector vehicle in a free flow traffic situation.

lengths could be adjusted. The approaches presented here could be part of a correction module for traffic state estimation until the penetration rate of ACVs allows a more complete overview of situations at intersections.

\section{Application}

The tests of the insertion method took place at the real-world intersection Tostmannplatz in Braunschweig, Germany (see Fig. 4). Only the insertion of vehicles based on CAMs was tested, not the applications (speed adaption and queue correction).

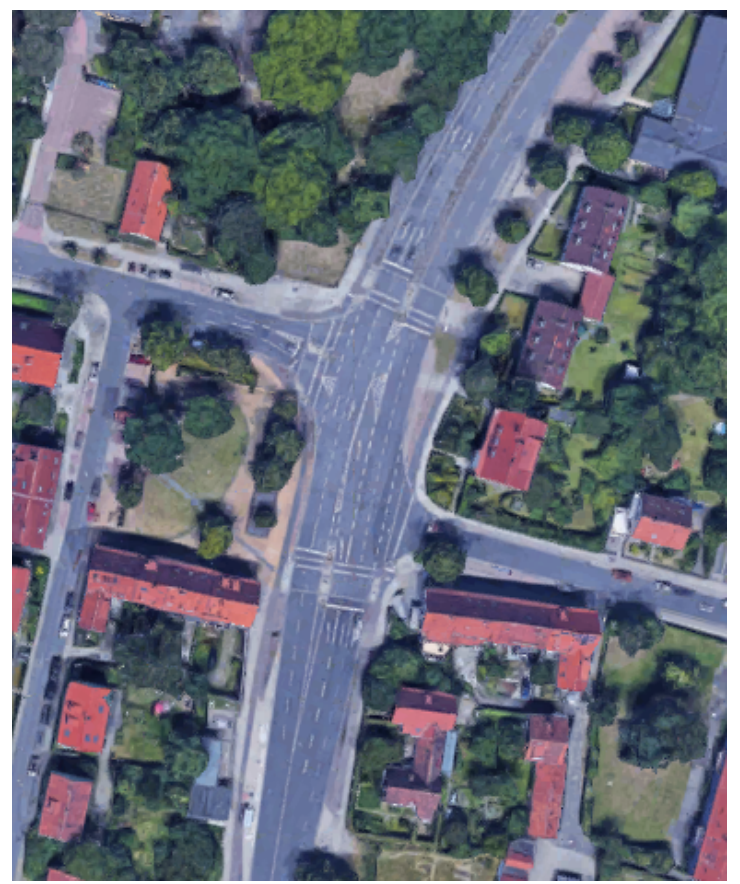

Figure 4: Aerial view of Tostmannplatz. (C)Google Maps

The intersection is part of $\mathrm{AIM}^{2}$, a test bed for automated and connected mobility in

${ }^{2}$ Application Platform for Intelligent Mobility, https://www.dlr.de/ts/aim\#gallery/25304 
Braunschweig operated by the DLR. It was already equipped with communication units (e.g. Road-Side-Unit (RSU) and Application Unit (AU)) that enable V2I communication. In the DLR project VITAL, the intersection was equipped with additional infrastructure to enable the operation of DLR traffic light control algorithms [10]. The new infrastructure consisted of two main components:

- Industrial computer: Running a SUMO simulation coupled with the AGLOSA algorithm (see [7]). The computer is connected to a traffic light controller that reacts to phase requests sent by the algorithm. In the other direction, the traffic controller passes its current phase to the simulation to synchronize the simulated and the real traffic light states. Also, information about detected vehicles is given into the simulation to generate a simulated traffic demand on which AGLOSA can plan.

- Magnet field sensors: Detect passing vehicles and transmit this information wirelessly to the traffic controller.

The insertion of CVs is done through the data provided by the magnet field sensors. This still is the main data source for inserting vehicles into the simulation. The insertion of vehicles by CAM is an extension to this functionality. To enable the processing of V2X information, the industrial computer was connected with the AU. Thus, incoming CAM messages can be passed to the simulation where the insertion method processes them into simulated vehicles.

The test of the insertion method took place during two separate test events in December 2018 and January 2019. During these events, the AGLOSA algorithm controlled the traffic light over the course of four hours. Two research vehicles were making test drives on Tostmannplatz broadcasting CAMs. These messages could successfully be processed into simulated vehicles and were taken into account by AGLOSA when planning the next phase sequence. Since the vehicles drove only in the main direction (northern and southern approaches) where more than one lane can be chosen by the simulated vehicles, the effect of the speed adaption could not be measured since the simulated vehicles took over the slower CAM generated cars.

\section{Conclusion}

This paper presented a method of inserting vehicles into a traffic simulation through V2X messages. It was shown on a real-world intersection that it is possible to feed V2X information into a traffic light control algorithm that already uses static detector information and to enrich the data base of the optimization procedure. Yet, the simulated vehicles in the test did not adapt to the speed of the CAM inserted vehicles since they were able to take over. This could be bypassed by driving CAM sending vehicles on single-lane approaches of the intersection or by ensuring a higher penetration rate of CAM sending vehicles.

Future work will focus on implementing and testing the speed adaption and queue length applications and measure their influences on the quality of the traffic flow.

\section{References}

[1] T. Williams, P. Alves, G. Lachapelle, C. Basnayake, "Evaluation of GPS-based methods of relative positioning for automotive safety applications", Transportation Research Part C: Emerging Technologies, Volume 23, 2012, Pages 98-108, ISSN 0968-090X, https://doi.org/10.1016/j.trc.2011.08.011. (http://www.sciencedirect.com/science/article/pii/S0968090X11001215) 
[2] D. Heß, S. Lapoehn, F. Utesch, M. Fischer, J. Schindler, T. Hesse, F. Köster, "Contributions of the EU Projects UnCoVerCPS and Enable-S3 to Highly Automated Driving in Conflict Situations", AAET 2019, February 5-7, Braunschweig

[3] J. Schindler, R. Dariani, M. Rondinone, T. Walter, "Dynamic and Flexible Platooning in Urban Areas", AAET Automatisiertes und vernetzes Fahren, March 14-15 2018, Braunschweig

[4] N. Wan, A. Vahidi, A. Luckow, Optimal speed advisory for connected vehicles in arterial roads and the impact on mixed traffic, Transportation Research Part C: Emerging Technologies, Volume 69, 2016, Pages 548-563, ISSN 0968-090X, https://doi.org/10.1016/j.trc.2016.01.011. (http://www.sciencedirect.com/science/article/pii/S0968090X16000292)

[5] D. Jia, D. Ngoduy, Enhanced cooperative car-following traffic model with the combination of V2V and V2I communication, Transportation Research Part B: Methodological, Volume 90, 2016, Pages 172-191, ISSN 0191-2615, https://doi.org/10.1016/j.trb.2016.03.008. (http://www.sciencedirect.com/science/article/pii/S0191261515302563)

[6] Y. Feng, K. Larry Head, S. Khoshmagham, M. Zamanipour, "A real-time adaptive signal control in a connected vehicle environment", Transportation Research Part C: Emerging Technologies, Volume 55, 2015, Pages 460-473, ISSN 0968-090X, https://doi.org/10.1016/j.trc.2015.01.007. (http://www.sciencedirect.com/science/article/pii/S0968090X15000091)

[7] J. Erdmann, "Combining Adaptive Junction Control with Simulatneous Green-Light-OptimalSpeed-Advisory", WiVec 2013, 2.-3. Juni 2013, Dresden, Deutschland. ISBN 9781467363358.

[8] European Telecommunications Standards Institute, "Intelligent Transport Systems (ITS); Vehicular Communications; Basic Set of Applications; Definitions", ETSI TR 102 638, European Telecommunications Standards Institute, June 2009.

[9] C. Priemer and B. Friedrich, "A Method For Tailback Approximation Via C2I-Data Based On Partial Penetration", in Proc. of the 15th World Congress on Intelligent Transport Systems, November 16-20, New York, 2008

[10] R. Oertel, J. Erdmann, R. Markowski, W. Schmidt, J. Trumpold, P. Wagner, "VITAL Verkehrsabhängig intelligente Steuerung von Lichtsignalanlagen". Straßenverkehrstechnik (09), Seiten 631-638. Forschungsgesellschaft für Straßen- und Verkehrswesen. ISSN 0039-2219 